\title{
High-energy QCD evolution from Slavnov-Taylor identity
}

\author{
Andrea Quadri* \\ INFN, Sez. di Milano and Dip. di Fisica, Univ. di Milano \\ via Celoria 16, I-20133 Milan \\ Italy \\ E-mail: andrea.quadri@mi.infn.it
}

We clarify the derivation of high-energy QCD evolution equations from the fundamental gauge symmetry of QCD. The gauge-fixed classical action of the Color Glass Condensate (CGC) is shown to be invariant under a suitable BRST symmetry, that holds after the separation of the gluon modes into their fast classical (background) part, the soft component and the semifast one, over which the one-step quantum evolution is carried out. The resulting Slavnov-Taylor (ST) identity holds to all orders in perturbation theory and strongly constrains the CGC effective field theory (EFT) arising from the integration of the soft modes. We show that the ST identity guarantees the gauge-invariance of the resulting EFT. It also allows to control the dependence on the gaugefixing choice for the semifast modes (usually the lightcone gauge in explicit computations). The formal properties of the evolution equations valid in different regimes (BKFL, JIMWLK, ...) can be all derived in a unified setting within this algebraic approach.

QCD-TNT-III-From quarks and gluons to hadronic matter: A bridge too far?,

2-6 September, 2013

European Centre for Theoretical Studies in Nuclear Physics and Related Areas (ECT*), Villazzano, Trento (Italy)

${ }^{*}$ Speaker. 


\section{Introduction}

The Color Glass Condensate (CGC) [1] is an effective field theory approach to the physics of high gluon densities and gluon saturation. The fast gluon modes (in the infinite momentum frame) are described by the classical solution to the Yang-Mills equation in the presence of some static color sources $\rho$. The latter are associated with a weight function $W_{\Lambda}[\rho]$, characterizing the CGC at the longitudinal scale $\Lambda$. Quantum Chromodynamics introduces radiative corrections around the classical (background) gluon configuration. These corrections affect the updated weight function $W_{b \Lambda}[\rho]$ at the new scale $b \Lambda$, with $b \ll 1$. Even when $\bar{\alpha}_{s} \ln 1 / b \ll 1$ (with $\bar{\alpha}_{s}=\alpha_{x} N / \pi, N$ being the number of colors), so that perturbation theory can be trusted, the quantum corrections give rise to logarithmically enhanced contribution that must be resummed through renormalization-group (RG) techniques. Depending on the different approximations used, one ends up with the wellknown BFKL [2]-[4] and JIMWLK evolution equations [5]-[11].

In this proceeding I would like to provide an introduction to some recent work aimed at clarifying the role of fundamental QCD gauge symmetry in constraining the properties of the effective field theory (EFT) of the CGC. The motivation is to separate the general features of the evolution equations, that only depend on the symmetry content of the model, from the specific aspects related e.g. to the choice of the gauge for the semifast modes or the particular approximation used in the computation of the EFT.

It turns out that such a program is indeed quite successful. The main results are the following. Gauge-invariance of the EFT (after the one-step quantum evolution) can be proven on the basis of the Slavnov-Taylor (ST) identity only. The ST identity encodes at the quantum level the classical BRST invariance of the QCD action. The ST identity also guarantees that the classical Yang-Mills equations of motion are not deformed by the quantum corrections. Thus the classical description of the CGC at the new scale $b \Lambda$ in terms of a modified weight function $W_{b \Lambda}[\rho]$, with the same equations of motion holding at the scale $\Lambda$, is indeed consistent. This is a crucial ingredient in the derivation of the evolution equations and is far from being obvious. It ultimately relies on the peculiar BRST symmetry of the CGC theory, after the separation of the gluons into their components (fast, semifast and soft ones).

The technical tools required for this program have been recently developed in a series of papers [12]-[14] where cohomological methods and generalized Lie series techniques have been used in order to constrain the complete background dependence in a gauge theory (for the full vertex functional and not only for its local approximation).

We will not try to sketch the proofs of the results for the CGC EFT here; details can be found in a forthcoming paper [15].

\section{Classical Action}

The classical action of the CGC is

$$
S_{C G C}[A, \rho]=S_{Y M}[A]+S_{W}[A, \rho],
$$

where we denote by $S_{Y M}$ the $S U(N)$ Yang-Mills action

$$
S_{Y M}=-\int d^{4} x \frac{1}{N} \operatorname{Tr}\left[F_{\mu v} F^{\mu v}\right]
$$


with $F_{\mu \nu}=F_{\mu \nu}^{a} T^{a}$ and $T^{a}$ the $S U(N)$ generators. In components the field strength reads $F_{\mu \nu}^{a}=$ $\partial_{\mu} A_{v}^{a}-\partial_{v} A_{\mu}^{a}+g f^{a b c} A_{\mu}^{b} A_{v}^{c} . f^{a b c}$ are the $S U(N)$ structure constants.

It is convenient to make use of the light-cone coordinates $x^{\mu}=\left(x^{+}, x^{-}, \mathbf{x}\right)$ with $x^{ \pm}=\left(x^{0} \pm\right.$ $\left.x^{3}\right) / \sqrt{2}$ and $\mathbf{x}=\left(x^{1}, x^{2}\right)$. We also use the vector notation $\vec{x}=\left(x^{-}, \mathbf{x}\right)$.

The source $\rho$ is the plus component (the only non-vanishing one) of the color current associated with the fast sources. In the infinite momentum frame $\rho=\rho(\vec{x})$ is static. The interaction with the gluon field is contained in $S_{W}$. Under the assumption that in lowest order the coupling is proportional to $\rho_{a}(\vec{x}) A_{a}^{-}(x)$ and by requiring gauge-invariance, $S_{W}$ turns out to be defined on some suitable Schwinger-Keldysh contour $C$ in the complex time plane, built by joining the path on the real axis from $-\infty$ to some $x_{f}^{+}$and backwards on a path from $x_{f}^{+}$to $-\infty$ with a small imaginary part and afterwards by taking the limit $x_{f} \rightarrow+\infty$. $S_{W}$ is given by [8]

$$
S_{W}[A, \rho]=\frac{i}{g N} \int d^{3} \vec{x} \operatorname{Tr}\left[p(\vec{x}) W_{C}(\vec{x})\right]
$$

where $W_{C}(\vec{x})$ is the contour temporal Wilson line

$$
W_{C}(\vec{x})=T_{C} \exp \left[i g \int_{C} d z A^{-}(z, \vec{x})\right] .
$$

In the infinite momentum frame (IMF) one can separate the fast gluon modes form the soft ones, i.e. we set

$$
A_{\mu}=\widehat{A}_{\mu}+a_{\mu}+\delta A_{\mu}
$$

$\widehat{A}_{\mu}$ represents the fast modes with longitudinal momenta $\left|p^{+}\right|>\Lambda$. It is fixed by the classical solution of the equation of motion of the CGC action $S_{C G C} . a_{\mu}$ describes the semi-fast modes with momenta $p^{+}$such that $\Lambda>\left|p^{+}\right|>b \Lambda$ (where we assume that $b \ll 1$, but $\bar{\alpha}_{s} \ln (1 / b) \ll 1$ ). The $a$-modes are the quantum fields that will be integrated out during the one-step quantum evolution. Finally, $\delta A_{\mu}$ are in turn the soft modes with momenta $\left|p^{+}\right|<b \Lambda$. They are fixed configurations during the one-step quantum evolution, so that the background actually has two components, a fast one $\left(\widehat{A}_{\mu}\right)$ and a soft one $\left(\delta A_{\mu}\right)$.

\section{BRST Symmetry}

The BRST symmetry of the full gluon field $A_{\mu}^{a}$ is obtained by replacing the gauge parameter with the Faddeev-Popov ghost field $C^{a}$, namely

$$
s A_{\mu}^{a}=D_{\mu}^{a b}[A] C^{b}, \quad D_{\mu}^{a b}[A]=\delta^{a b} \partial_{\mu}+g f^{a c b} A_{\mu}^{c}
$$

The background $\widehat{A}_{\mu}^{a}$ is paired into a BRST doublet [16], [17] through

$$
s \widehat{A}_{\mu}^{a}=\Omega_{\mu}^{a}, \quad s \Omega_{\mu}^{a}=0 .
$$

$\Omega_{\mu}^{a}$ is an anticommuting external source with ghost number one. Eq.(3.2) guarantees that the cohomology of the theory [17] (and hence the physical observables) is unaffected by the introduction of the background configuration. In the standard approach to the algebraic treatment of the Background Field Method [18]-[20], Eq.(3.1) together with Eq.(3.2) fixes uniquely the BRST 
transformation of the quantum field $Q_{\mu}=A_{\mu}-\widehat{A}_{\mu}$. In the present case there is one more field in the game, namely $\delta A$. However in the CGC prescription for obtaining the EFT, $\delta A$ plays the role of a background field and thus cannot have a $\Omega$-dependent term in its BRST variation. It is also convenient to split the ghost $C^{a}$ into a soft $\left(\delta c^{a}\right)$ and a semifast $\left(c^{a}\right)$ component according to

$$
C^{a}=c^{a}+\delta c^{a}
$$

This leads us to the following BRST transformations for the gluon modes:

$$
s \delta A_{\mu}^{a}=D_{\mu}^{a b}[\delta A] \delta c^{b}, \quad s a_{\mu}^{a}=s A_{\mu}^{a}=s \delta A_{\mu}^{a}-s \widehat{A}_{\mu}^{a} .
$$

In the presence of a soft component of the gluon field that is not integrated out, the color current entering into $S_{W}$ must be evaluated by using the Wilson line from $z^{+} \rightarrow-\infty$ to $x^{+}$of the soft modes $\delta A^{-}$[15]. The classical equation of motion then becomes (we denote by $\alpha$ the plus component of the fast background field, that can be chosen to be the only non-vanishing one in the Coulomb gauge):

$$
\nabla_{x}^{2} \alpha(x)=-U^{\dagger}(x) J^{+}(x) U(x)
$$

where $J^{+}$is the plus component of the color-rotated current and $U^{\dagger}$ is the Wilson line

$$
U^{\dagger}(x)=\operatorname{Pexp}\left[\mathrm{ig} \int_{-\infty}^{x^{-}} \mathrm{d} z^{-} \alpha\left(x^{+}, z^{-}, x\right)\right],
$$

It is convenient to redefine the source in the r.h.s. of Eq.(3.5) by setting

$$
\chi(x) \equiv U^{\dagger}(x) J^{+}(x) U(x) .
$$

Then, $\chi$ becomes the independent variable; it transforms in the adjoint representation of SU(N), and therefore

$$
s \chi^{a}=g f^{a b c} \chi^{b} \delta c^{c}
$$

Again one has to split $\chi$ into a background part and a quantum fluctuation

$$
\chi^{a}=\widehat{\chi}^{a}+\delta \chi^{a}
$$

$\widehat{\chi}^{a}$ is the source associated to the classical field $\alpha$ and hence its BRST transformation is given by

$$
s \widehat{\chi}^{a}=-\nabla_{x}^{2} \Omega^{a+}(x),
$$

while the BRST transformation of $\delta \chi$ is obtained by difference from Eq.(3.8) and Eq.(3.9).

\section{ST identity for the EFT}

The quantization of the theory proceeds by choosing a gauge-fixing for the semifast modes. This is usually done in the lightcone gauge, where ghosts decouple. This is why they never appear in explicit computations in the CGC framework. However, having introduced the appropriate BRST symmetry, nothing prevents to choose a different gauge. The ghost interactions are automatically fixed by the BRST symmetry in the usual fashion and one must of course include their contributions 
in the computation of the effective action at leading order in $\bar{\alpha}_{s} \ln (1 / b)$. This is discussed in detail in [15].

The existence of a classical BRST-invariant action allows one to write in the usual way [21] the Slavnov-Taylor (ST) identity for the full vertex functional $\Gamma$, valid to all orders in the loop expansion if the theory is non-anomalous (as it happens for QCD).

In the CGC framework one is not really interested in $\Gamma$, but in the effective action $\widetilde{\Gamma}$ obtained by intgrating out the semifast modes $a_{\mu} . \widetilde{\Gamma}$ is the generating functional of all diagrams that are one-particle reducible (1-PR) with respect to (w.r.t.) $a_{\mu}$ and one-particle irreducibile (1-PI) w.r.t. all other fields. We will not dwell here on the details of the derivation of $\tilde{\Gamma}$ and just report the ST identity that it fulfills:

$$
\begin{aligned}
\mathscr{S} \widetilde{\Gamma} \equiv \int \mathrm{d}^{4} z & {\left[\Omega_{\mu}^{a}(z) \frac{\delta \widetilde{\Gamma}}{\delta \widehat{A}_{\mu}^{a}(z)}+\frac{\delta \widetilde{\Gamma}}{\delta\left(\delta A_{\mu}^{* a}(z)\right)} \frac{\delta \widetilde{\Gamma}}{\delta\left(\delta A_{\mu}^{a}(z)\right)}+\frac{\delta \widetilde{\Gamma}}{\delta\left(\delta c^{* a}(z)\right)} \frac{\delta \widetilde{\Gamma}}{\delta\left(\delta c^{a}(z)\right)}+\delta b^{a}(z) \frac{\delta \widetilde{\Gamma}}{\delta\left(\delta \bar{c}^{a}(z)\right)}\right.} \\
& \left.+\frac{\delta \widetilde{\Gamma}}{\delta\left(\delta \chi^{* a}(z)\right)} \frac{\delta \widetilde{\Gamma}}{\delta\left(\delta \chi^{a}(z)\right)}\right]=0 .
\end{aligned}
$$

In the equation above, $\delta \bar{c}^{a}$ is the antighost for the soft modes and $\delta b^{a}$ the corresponding NakanishiLautrup multiplier field. $\delta A^{*}, \delta c^{*}$ and $\delta \chi^{*}$ are the antifields (i.e. the sources of the BRST transformations) of the soft gluons, the soft ghost and the quantum fluctuations of the color sources $\chi$ respectively.

\subsection{Gauge invariance}

Eq. (4.1) yields very strong constraints on the effective action of the CGC. Let us take a derivative w.r.t. $\delta c^{b}$ and then set $\delta c, \Omega$ and $\delta b$, to zero. One finds

$$
\int \mathrm{d}^{4} z\left[\frac{\delta^{2} \widetilde{\Gamma}}{\delta\left(\delta c_{b}(x)\right) \delta\left(\delta A_{\mu}^{* a}(z)\right)} \frac{\delta \widetilde{\Gamma}}{\delta\left(\delta A_{\mu}^{a}(z)\right)}+\frac{\delta^{2} \widetilde{\Gamma}}{\delta\left(\delta c_{b}(x)\right) \delta\left(\delta \chi_{\mu}^{* a}(z)\right)} \frac{\delta \widetilde{\Gamma}}{\delta\left(\delta \chi_{\mu}^{a}(z)\right)}\right]=0 .
$$

At $\Omega=0$ the BRST variations $s \delta A$ and $s \delta \chi$ contain neither $a$ nor $c$ and hence do not receive radiative corrections upon the integration of the semifast modes. This means that they remain classical, namely

$$
\begin{aligned}
& \frac{\delta^{2} \widetilde{\Gamma}}{\delta\left(\delta c_{b}(x)\right) \delta\left(\delta A_{\mu}^{* a}(z)\right)}=\frac{\delta^{2} \widetilde{\Gamma}^{(0)}}{\delta\left(\delta c_{b}(x)\right) \delta\left(\delta A_{\mu}^{* a}(z)\right)}=\delta^{a b} \partial_{\mu} \delta^{(4)}(x-z)+g f^{a c b} \delta A_{\mu}^{c} \delta^{(4)}(x-z), \\
& \left.\frac{\delta^{2} \widetilde{\Gamma}}{\delta\left(\delta c_{b}(x)\right) \delta\left(\delta \chi^{* a}(z)\right)}\right|_{\Omega=0}=\left.\frac{\delta^{2} \widetilde{\Gamma}^{(0)}}{\delta\left(\delta c_{b}(x)\right) \delta\left(\delta \chi^{* a}(z)\right)}\right|_{\Omega=0}=g f^{a c b}\left[\widehat{\chi}^{c}(x)+\delta \chi^{c}(x)\right] \delta^{(4)}(x-z)
\end{aligned}
$$

Hence Eq. (4.2) amounts to the statement of gauge invariance of the effective action $\widetilde{\Gamma}$. We stress that this result holds irrespectively of the gauge choice for the semi-fast modes: that is, gauge invariance follows as a consequence of the ST identity (4.1) and of the semifast-soft decomposition. 


\section{5. (Quantum-deformed) Background Equations of Motion}

Taking a functional differentiation of Eq. (4.1) w.r.t. the source $\Omega$, and setting $\Omega=0$ afterwards, yields (at $\delta b=0$ )

$$
\frac{\delta \widetilde{\Gamma}}{\delta \widehat{A}_{\mu}^{a}(x)}=-\int \mathrm{d}^{4} z\left[\frac{\delta \widetilde{\Gamma}}{\delta \Omega_{\mu}^{a}(x) \delta\left(\delta A_{v}^{* b}(z)\right)} \frac{\delta \widetilde{\Gamma}}{\delta\left(\delta A_{\mu}^{a}(z)\right)}+\frac{\delta \widetilde{\Gamma}}{\delta \Omega_{\mu}^{a}(x) \delta\left(\delta \chi^{* b}(z)\right)} \frac{\delta \widetilde{\Gamma}}{\delta\left(\delta \chi^{b}(z)\right)}\right] .
$$

This is a fundamental equation for the effective field theory, as it encodes how quantum fluctuations will modify the classical equation of motion (3.5) for the background field configuration.

The first term in (5.1) controls the (gauge-dependent) deformation of the classical backgroundquantum splitting induced by quantum corrections [12]-[14]. In general (e.g. for gauge theories in the presence of instanton configurations) this deformation is not zero.

The second term fixes instead the functional dependence of the background $\widehat{A}$ on the color charge density $\delta \chi$, once quantum corrections are taken into account.

This result is very general: it does not rely on the specific form of the action chosen, on the gauge-fixing adopted for the semifast modes, and on the order of approximation used in order to compute radiative corrections.

Let us now specialize to the CGC case. By taking into account the structure of the BRST symmetry it can be proven [15] that

$$
\widetilde{\Gamma}_{\Omega_{a}^{\mu} \delta A_{b}^{* v}}(x, y)=0
$$

while

$$
\widetilde{\Gamma}_{\Omega_{a}^{\mu} \delta \chi_{b}^{*}}(x, y)=\Gamma_{\Omega_{a}^{\mu} \delta \chi_{b}^{*}}^{(0)}(x, y)=-\delta^{\mu+} \nabla_{x}^{2} \delta^{(4)}(x-y) .
$$

It is understood that the background field has not been set to zero here, so Eqs.(5.2) and (5.3) are valid in the presence of the background $\alpha$. Eq.(5.2) tells us that the equation of motion for the background will not be affected by the soft modes of the theory. This is physically reassuring, since it means that the separation of scales is preserved under the quantum evolution. Eq.(5.3) in turn implies that the equation of motion, relating the background field to the color sources, maintains the same classical form after quantum corrections are taken into account. This is a non-trivial result that follows directly from the symmetry properties of the theory. It is a crucial property in order to be able to encode all the effects of the quantum fluctuations into an updated weight function $W_{b \Lambda}[\rho]$, while determining the new background configuration again by the same classical equation of motion valid for the theory at the scale $\Lambda$.

\section{Conclusions}

Gauge invariance of the CGC effective action, as well as the stability of the background equation of motion under the one-step quantum evolution, follow from basic symmetry properties of the theory, encoded in the ST identity. In particular it does not depend on the choice of the semifast modes and on the order of approximation used in the evaluation of the effective action. The general structure of the evolution equations can also be derived within this purely algebraic setting [15]. 


\section{Acknowledgments}

The author wishes to acknwoledge the warm hospitality and the support of ECT* during the workshop.

\section{References}

[1] See e.g. F. Gelis, E. Iancu, J. Jalilian-Marian and R. Venugopalan, Ann. Rev. Nucl. Part. Sci. 60 (2010) 463 [arXiv:1002.0333 [hep-ph]].

[2] L. N. Lipatov, Sov. J. Nucl. Phys. 23 (1976) 338 [Yad. Fiz. 23 (1976) 642].

[3] E. A. Kuraev, L. N. Lipatov and V. S. Fadin, Sov. Phys. JETP 45 (1977) 199 [Zh. Eksp. Teor. Fiz. 72 (1977) 377].

[4] I. I. Balitsky and L. N. Lipatov, Sov. J. Nucl. Phys. 28 (1978) 822 [Yad. Fiz. 28 (1978) 1597].

[5] J. Jalilian-Marian, A. Kovner, A. Leonidov and H. Weigert, Nucl. Phys. B 504 (1997) 415 [hep-ph/9701284].

[6] J. Jalilian-Marian, A. Kovner, A. Leonidov and H. Weigert, Phys. Rev. D 59 (1998) 014014 [hep-ph/9706377].

[7] A. Kovner, J. G. Milhano and H. Weigert, Phys. Rev. D 62 (2000) 114005 [hep-ph/0004014].

[8] E. Ferreiro, E. Iancu, A. Leonidov and L. McLerran, Nucl. Phys. A 703 (2002) 489 [hep-ph/0109115].

[9] E. Iancu, A. Leonidov and L. D. McLerran, Phys. Lett. B 510 (2001) 133 [hep-ph/0102009].

[10] E. Iancu, A. Leonidov and L. D. McLerran, Nucl. Phys. A 692 (2001) 583 [hep-ph/0011241].

[11] H. Weigert, Nucl. Phys. A 703 (2002) 823 [hep-ph/0004044].

[12] D. Binosi and A. Quadri, Phys. Rev. D 84 (2011) 065017 [arXiv:1106.3240 [hep-th]].

[13] D. Binosi and A. Quadri, Phys. Rev. D 85 (2012) 085020 [arXiv:1201.1807 [hep-th]].

[14] D. Binosi and A. Quadri, Phys. Rev. D 85 (2012) 121702 [arXiv:1203.6637 [hep-th]].

[15] D. Binosi, A. Quadri, D. Triantafyllopoulos, in preparation.

[16] A. Quadri, JHEP 0205 (2002) 051 [hep-th/0201122].

[17] G. Barnich, F. Brandt and M. Henneaux, Phys. Rept. 338 (2000) 439 [hep-th/0002245].

[18] P. A. Grassi, Nucl. Phys. B 462 (1996) 524 [hep-th/9505101].

[19] R. Ferrari, M. Picariello and A. Quadri, Annals Phys. 294 (2001) 165 [hep-th/0012090].

[20] C. Becchi and R. Collina, Nucl. Phys. B 562 (1999) 412 [hep-th/9907092].

[21] J. Gomis, J. Paris and S. Samuel, Phys. Rept. 259 (1995) 1 [hep-th/9412228]. 